\title{
Role of electronic excitation on the anomalous magnetism of elemental Copper
}

\author{
Sudip Pal, Sumit Sarkar, Kranti Kumar, R. Raghunathan, \\ R. J. Choudhary, A. Banerjee, and S. B. Roy* \\ UGC-DAE Consortium for Scientific Research, \\ University Campus, Khandwa Road, \\ Indore- 452001, India
}

\begin{abstract}
Magnetic susceptibility of elemental copper $(\mathrm{Cu})$ shows an anomalous rise at low temperatures superimposed on the expected atypical diamagnetic response. Such temperature dependent susceptibility, which is also known as the Curie tail, can not be explained on the basis of Larmor diamagnetic and Pauli paramagnetic contributions expected in $\mathrm{Cu}$. Using valence band resonant photoemission spectroscopy results and density functional theory calculations, we show the magnetic anomaly appears due to presence of holes in $\mathrm{Cu} 3 d$ band, which originates from thermally excited electronic configuration. Our study therefore highlights that the Curie tail, which is generally overlooked presuming it either due to paramagnetic impurities or defects, can in fact be intrinsic to a material, and even simple systems like elemental $\mathrm{Cu}$ is susceptible to electronic excitations giving rise to anomalous magnetic state.
\end{abstract}

* sudip.pal111@gmail.com, sbroy@csr.res.in 


\section{INTRODUCTION}

Research on magnetism for the past several decades, has been devoted to understanding the transition between a disordered paramagnetic and an ordered magnetic ground state. Interestingly, several materials exhibit an unexpected upturn in the magnetic susceptibility at very low temperatures (typically below $50 \mathrm{~K}$ ) without any subsequent phase transition to an ordered magnetic state. This anomalous upturn is often termed as the Curie tail and is generally attributed to an extrinsic origin like the presence of paramagnetic impurities or defects resulting from the synthesis process [1 $[8]$. This anomaly is now becoming more common in a wide range of complex materials. For example, such behavior has recently been found in exotic quantum spin liquids like $\mathrm{Sr}_{3} \mathrm{CuSb}_{2} \mathrm{O}_{9}$ and $1 \mathrm{~T}-\mathrm{TaS}_{2}$ as well as topological materials like $\mathrm{TaSe}_{3}$ [9, 10]. So, it is important to carefully probe and microscopically understand this anomalous magnetic behaviour. This will reveal whether the Curie tail always has an extrinsic origin or in some cases it can be intrinsic to the material.

In compounds, the complex atomic and electronic structures make it difficult to clearly understand the intrinsic mechanisms that govern the overall magnetic properties. In this context, elemental metal like copper can be a very suitable starting point. Elemental copper has the electronic configuration $\mathrm{Cu}$ : $[A r] 3 d^{10} 4 s^{1}$, and hence we would expect only two contributions to the overall magnetic susceptibility namely, the diamagnetism from the completely filled $3 d$ orbital and a Pauli paramagnetic contribution of the half filled $4 s$ electrons [11 13]. As both these contributions are temperature independent, the overall magnetic susceptibility of $\mathrm{Cu}$ should also be independent of temperature. But, few decades back, studies on the magnetic behavior of about $99.99 \%$ pure elemental copper $(\mathrm{Cu})$ have shown anomalous temperature dependence of magnetic susceptibility, indicating the presence of an additional but unusual contribution at low temperatures [14, 15]. Here, we show that the magnetic susceptibility of elemental $\mathrm{Cu}$ is indeed temperature dependent at low temperatures. However, contrary to the conventional wisdom that the Curie tail is related to the presence of paramagnetic impurity, it originates from the electronic excitations.

We have recorded the temperature $(T)$ and magnetic field $(H)$ dependent magnetic response of $99.99 \%$ pure elemental $\mathrm{Cu}$. Using x-ray flourescence (XRF) measurements we

found that the standard copper sample contains about $0.01 \%$ transition metal impurity which can potentially lead to a paramagnetic tail at low temperature. However, even with 


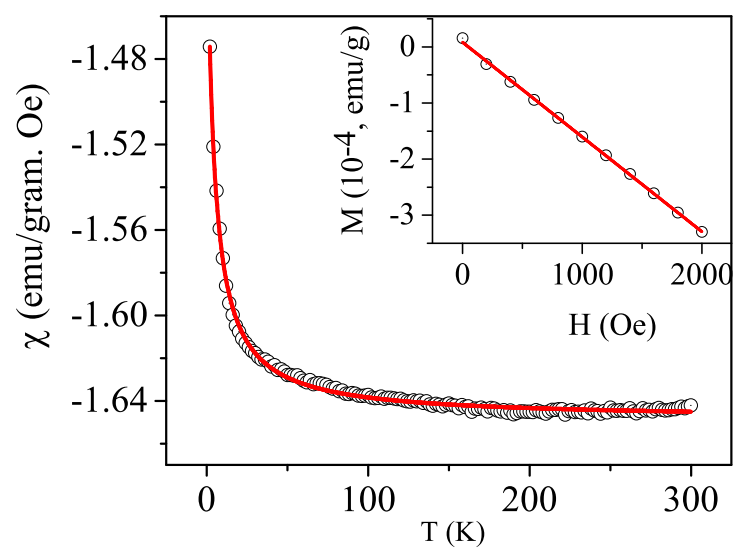

FIG. 1. $\chi$-T curve measured at $\mathrm{H}=2 \mathrm{kOe}$ while cooling the sample. Inset shows the M-H curve at $T=300 \mathrm{~K}$. Red lines are fit to the data points. See text for details.

such paramagnetic impurity, the overall magnetic behaviour can not be accounted. Our valence band and resonant photoemission spectroscopy reveal the presence of hole in the $3 d$ band. This creates an intrinsic spin moment $\left(s=\frac{1}{2}\right)$ on a fraction of copper atoms giving rise to an additional Curie-like paramagnetic contribution. Importantly, the concentration of holes reduces with decrease in temperature. Our results thus show that the observed anomalous magnetic behaviour of copper is intrinsic in nature.

\section{METHODS}

Magnetic measurements were carried out in a Superconducting Quantum Interference Device (SQUID) magnetometer (M/S Quantum Design, USA). The resonating valence band spectra (VBS) were recorded at different photon energy values in the range of $60-76 \mathrm{eV}$ using the angle integrated photoemission spectroscopy (AIPES) beamline at the Indus-1 synchrotron source at RRCAT, Indore, India. XRF measurement has been performed at XRF beamline on Indus-2 synchrotron radiation source at RRCAT. The results of this XRF study revealed the presence of $0.01 \% \mathrm{Mn}$ impurity in our $\mathrm{Cu}$ sample. Density of states (DOS) has been calculated within the framework of Density functional theory (DFT) by employing PAW potentials using the Vienna Ab-initio Simulation Package (VASP) [16, 17].

The main panel of Fig. 1 shows the variation of dc susceptibility with temperature at $H=2 \mathrm{kOe}$. The susceptibility at $300 \mathrm{~K}$ is $-1.64 \times 10^{-7} \mathrm{emu} /(\mathrm{g}$. Oe), and ensures 

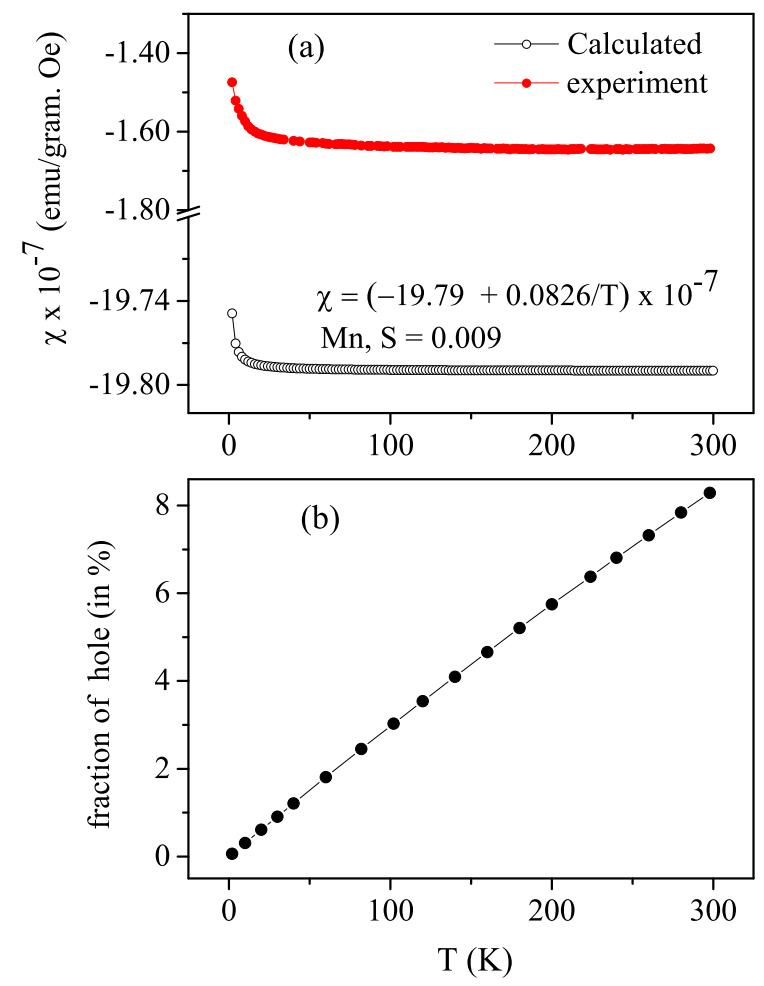

FIG. 2. (a) It shows the experimental and calculated $\chi-T$ data. The calculated temperature variation of $\chi$ has been obtained by considering the diamagnetic and Pauli paramagnetic contribution of $\mathrm{Cu}$ and Curie-Weiss paramagnetic contribution of $0.01 \%$ of $\mathrm{Mn}$ present in the sample. (b) shows fraction of hole estimated by using eqn. 5 from the additional susceptibility observed in experiment, as compared to the calculated value.

the dominating diamagnetic nature of the sample. In the isothermal $M-H$ curve at $T=300 \mathrm{~K}$ (inset of Fig. 1) $M$ is negative and varies linearly with $\mathrm{H}$, which reveals a predominant diamagnetic nature of the $\mathrm{Cu}$ sample. The dc susceptibility $(\chi=M / H)$ at $300 \mathrm{~K}$, obtained from the linear fit of the $M-H$ curve is $\chi=-1.66 \times 10^{-7} \mathrm{emu} /(\mathrm{g}$.Oe). A diamagnetic sample should show a constant negative value of susceptibility over the entire temperature range. However, our experimental results indicate that $M$ is nearly constant down to $150 \mathrm{~K}$ and below this temperature, $M$ gradually increases with further decrease in temperature and finally increases rapidly below $15 \mathrm{~K}$. In the remainder of this discussion, we refer to this Curie-Weiss paramagnetic like contribution below $150 \mathrm{~K}$ as the Curie tail. For an elemental metal like copper with a completely filled $3 d$ and an half-filled $4 s$ shells, we expect diamagnetic and Pauli paramagnetic contributions respectively. As both these contributions should be temperature independent, the low-temperature Curie tail is rather 
surprising. Such temperature dependence of magnetization is qualitatively similar to those reported earlier [14, 15]. The susceptibility can be fitted considering additional Curie-Weiss like term $\left(\frac{C}{\chi-\chi_{0}}\right)$ as shown in Fig. 1 (red line), where $C$ is a constant giving us the value of the impurity spin. Even though the magnetic data can be fitted with the Curie-Wiess law, we will show in the following sections that such fitting would be meaningless, because $C$ is actually temperature dependent due to the intrinsic property of copper.

In order to understand this anomalous magnetic behaviour, we analytically calculate the diamagnetic and Pauli paramagnetic contributions to the total magnetic susceptibility. In case of elemental $\mathrm{Cu}$ with atomic number $Z=29$, the conventional electronic configuration is $[A r] 3 d^{10} 4 s^{1}$. The Larmor diamagnetic contribution can be estimated from, [11, 12]

$$
\chi_{D}=-0.79 Z_{i} \times 10^{-6}\left\langle\left(r / a_{0}\right)\right\rangle^{2} \mathrm{emu} /(\mathrm{g} . \mathrm{Oe})
$$

Here, $Z_{i}$ is the number of electrons in the atom, $r$ is the atomic radius (for $\mathrm{Cu}, r=1.28 \AA$ ) and $a_{0}$ is the Bohr radius. Taking $\left\langle\left(r / a_{0}\right)\right\rangle=2.42$, diamagnetic susceptibility can be estimated as $\chi_{D}=-20.30 \times 10^{-7} \mathrm{emu} /\left(\mathrm{g}\right.$.Oe). In addition to this $\chi_{D}$, conduction electrons give rise to a positive magnetic susceptibility, known as the Pauli paramagnetism [11, 13]. At $T=0$ $\mathrm{K}$, the Pauli paramagnetic susceptibility can be written as,

$$
\chi_{P}=\mu_{B}^{2} \times g\left(E_{F}\right)
$$

where, $g\left(E_{F}\right)$ is the total density of states at the Fermi level, which can be calculated using DFT. In case of $\mathrm{Cu}$, it is obtained to be $0.10 \mathrm{eV}^{-1}$ (see Fig. 3 inset). Substituting this in Eqn. 2 gives the Pauli paramagnetic susceptibility, $\chi_{P}=0.51 \times 10^{-7} \mathrm{emu} /(\mathrm{g}$.Oe). It may be noted that at finite temperature, smearing of the Fermi surface introduces a very small correction to eqn. 2. However, in case of $\mathrm{Cu}$, due to very large Fermi temperature (around $80,000 \mathrm{~K}$ ), this correction is negligible up to room temperature. Now, the net susceptibility can be obtained by summing the dia- and para-magnetic contributions $\left(\chi=\chi_{D}+\chi_{P}\right)$ which gives, $\chi=-19.79 \times 10^{-7} \mathrm{emu} /(\mathrm{g}$.Oe). Our calculated total susceptibility is one order of magnitude larger than the experimental data, suggesting the presence of additional contributions apart from $\chi_{D}$ and $\chi_{P}$, and this warrants an explanation.

The results of our XRF measurements suggested the presence of about $0.01 \%$ of $\mathrm{Mn}$ impurity in the present $\mathrm{Cu}$ sample. Therefore, it would be instructive to understand the effect of electron delocalisation on effective moment of a Mn atom with an electron configuration $[A r] 3 d^{5} 4 s^{2}$ in a copper lattice. So, we performed DFT calculations on a $5 \times 5 \times 5$ 


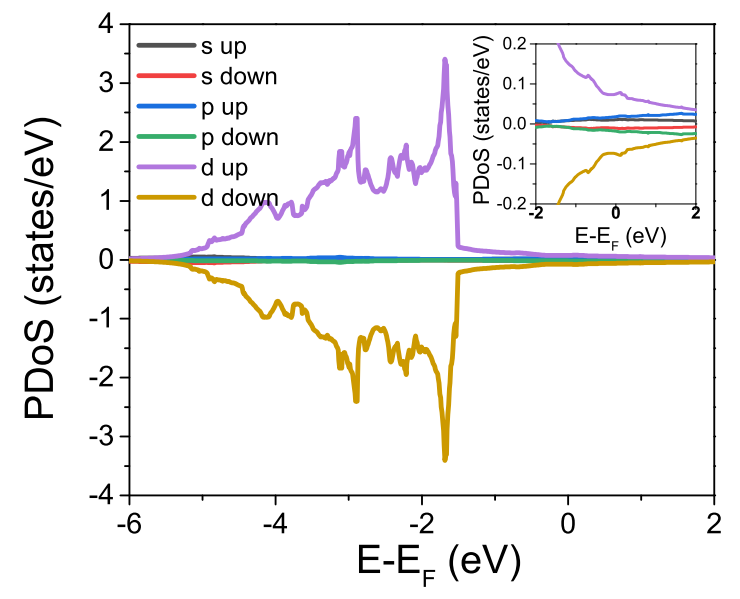

FIG. 3. Partial density of states near $E_{F}$ at $T=0$ obtained from DFT. At $E_{F}$, total DoS is 0.1 $\mathrm{eV}^{-1}$

supercell of copper containing 500 atoms. For the pure copper case, our calculations show that the copper atoms have a negligible magnetic moment of $0.02 \mu_{B}$. We also carried out DFT calculations of this supercell by replacing a copper atom with a manganese atom. This corresponds to an effective impurity concentration of $0.2 \%$. It should be noted here that our goal here is not to mimic the experimental impurity concentration, but rather to identify the role of electron delocalisation on the magnetic moment of copper and manganese atoms. Our calculations show that the presence of manganese also enhances the moment of copper atoms to $0.06 \mu_{B}$, but still the copper moment is negligible. The magnetic moment of manganese is equivalent to spin, $S_{M n}=0.009$, much lesser compared to the expected $S=\frac{5}{2}$ for five unpaired electrons.

Now that we know the magnetic moments of manganese, we can add the paramagnetic contribution using the Curie-Weiss relation with the diamagnetic and Pauli paramagnetic contributions to obtain the total magnetic susceptibility $\chi=\chi_{D}+\chi_{P a u l i}+\chi_{P M}^{M n}(T)$.

$$
\begin{array}{r}
\chi_{P M}^{M n}(T)=\frac{N_{A} g^{2} \mu_{B}^{2} S_{M n}\left(S_{M n}+1\right)}{3 k_{B} T} \\
=\frac{0.0826 \times 10^{-7}}{T} \mathrm{emu} / \text { gram. Oe }
\end{array}
$$

Here, $S_{M n}$ corresponds to the Mn spin moment, $N_{A}$ is the Avagadro number, $\mu_{B}$ is the Bohr magneton, $g$ is the gyromagnetic ratio which is taken to be $2, k_{B}$ is the Boltzman constant and $T$ is the temperature. The calculated susceptibility is plotted as a function of temperature in Fig. 2(a), together with the experimental data. Note that, the addition of 

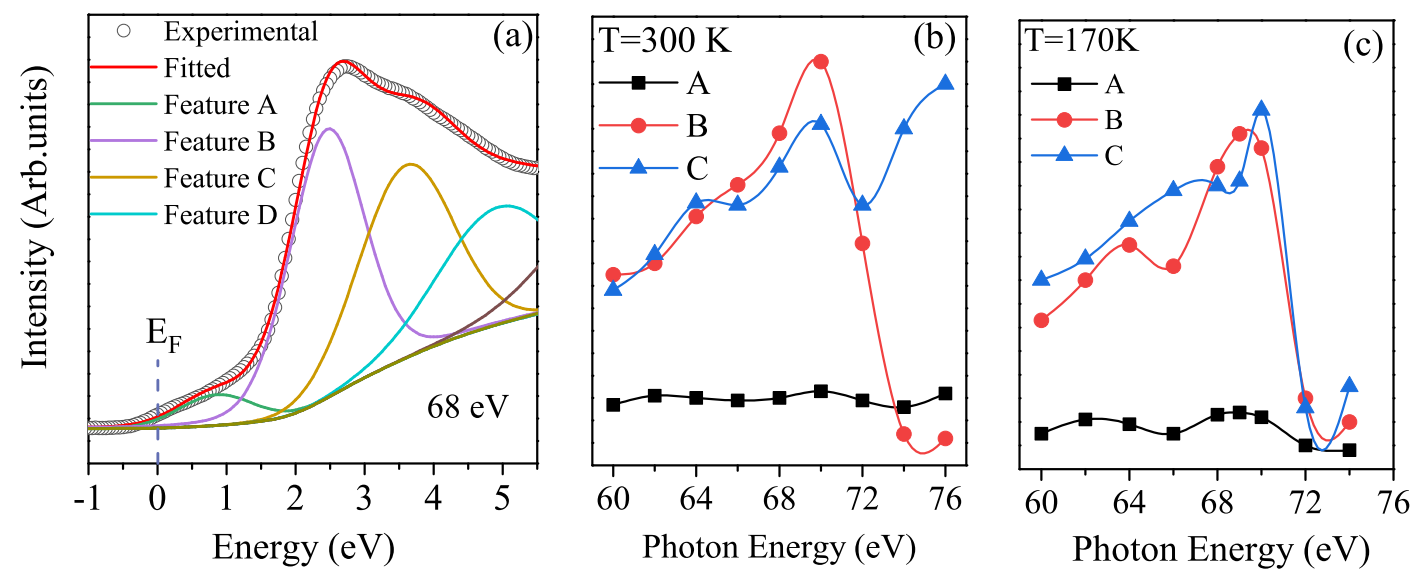

FIG. 4. (a) Experimental data of RPES at $T=300 \mathrm{~K}$ at the incident photon energy of $68 \mathrm{eV}$ fitted by considering Gaussian shape peaks and Shirley background, (b) and (c) show CIS plot of the near $E_{F}$ spectral features at $T=300$ and $170 \mathrm{~K}$ respectively obtained from the fitting of the valance band measured at each incident photon energies.

0.01\% Mn impurity can not reproduce the experimentally observed susceptibility behaviour, which suggests the presence of additional contribution which is perhaps intrinsic to copper.

Generally in $3 d$ transition metals, the $3 d$ and $4 s$ orbital energies are close enough. This allows for electrons to exist in two possible electronic states. Our conventional wisdom suggests that copper exists in the $[A r] 3 d^{10} 4 s^{1}$ configuration, whereas energetically $[A r] 3 d^{9} 4 s^{2}$ electronic state is also possible. At absolute zero, the Fermi-Dirac function is a step function with all the states below the Fermi level are filled and all those states above $E_{F}$ are empty. This corresponds to the electron configuration $[A r] 3 d^{10} 4 s^{1}$. At temperatures above absolute zero, due to thermal energy some of the electrons can get excited from the $3 d$ to $4 s$ band. This hypothesis is further supported by our partial DOS calculated using DFT which is presented in Fig. 3. We notice that the DOS weights for the $3 d$ band is non-zero even above the Fermi level. These observations suggest that the $3 d$ band of copper potentially has holes. This can open up the possibility of unpaired electrons in the $3 d$ band leading to intrinsic magnetic moments on a fraction of copper sites due to the instability created by fluctuation between $[A r] 3 d^{10} 4 s^{1} \Leftrightarrow[A r] 3 d^{9} 4 s^{2}$ electronic states, due to the thermal energy. The $3 d^{10} 4 s^{1}$ configuration of copper gives diamagnetic plus Pauli paramagnetic configuration, whereas a $3 d^{9} 4 s^{2}$ configuration would result in a combination of paramagnetic and diamagnetic configuration. Therefore, the magnetic property of copper is governed by the competition 
between these two sets of contributions:

$$
\chi_{D}+\chi_{P P M} \Leftrightarrow \chi_{P M}+\chi_{D}
$$

In the light of holes in the $3 d$ band, we now discuss the observed temperature dependence of magnetic susceptibility in two temperature regimes: $(i) T>150 \mathrm{~K}$ and $(i i) T<150 \mathrm{~K}$. In region $(i)$, say $300 \mathrm{~K}$, there will be significant number of holes in the $3 d$ band leading to larger concentration of paramagnetic ions. The smearing of electrons around the Fermi level decreases as the temperature is lowered. Correspondingly, the concentration of paramagnetic copper atoms due to the intrinsic moment of copper also decreases. As the paramagnetic susceptibility is inversely related to the temperature, the overall susceptibility remains more or less constant in this temperature range. But this also enhances the diamagnetic contribution of $3 d$ electrons, but this is few orders of magnitude smaller than the paramagnetic contribution. The temperature dependence of susceptibility in regime ( $i i)$ shows a Curie tail. In this temperature regime, the paramagnetic contributions both from the Mn impurity and the intrinsic moment of copper become dominant as the temperature is lowered. At the same time, as the Fermi smearing reduces with decrease in temperature, the concentration of intrinsic moment also decreases. However, the final behaviour will depend upon the rate at which the concentration of intrinsic moment decreases with temperature. Typically at temperatures close enough to absolute zero, the electron configuration of copper would tend to $[A r] 3 d^{10} 4 s^{1}$ and the Curie tail would be governed solely by the Mn impurity. With this argument, the total susceptibility of copper can be written as follows.

$$
\begin{aligned}
\chi(T)= & \chi_{D}+[1+x(T)] \chi_{P P M} \\
& +\chi_{P M}^{M n}(T)+x(T) \chi_{P M}^{C u}(T)
\end{aligned}
$$

The terms in the above equation correspond to contributions from diamagnetism, Pauli paramagnetism, paramagnetism due to Mn moments and paramagnetism due to $\mathrm{Cu}$ moments respectively. The factor $x(T)$ is the concentration of $\mathrm{Cu}$ moments at temperature T due to the Fermi smearing. The total susceptibility versus temperature calculated thus can be matched to the experimental data to obtain the fraction of holes $x(T)$ at different temperatures, which is shown in Fig. 2(b). As expected, due to the decrease in smearing, the concentration of $\mathrm{Cu}$ moments $x(T)$ decreases as the temperature is decreased.

In order to further validate our hypothesis of existence of intrinsic copper moments due to the presence of holes in $3 d$ band, we recorded the valance band spectrum (VBS) in the 
vicinity of Fermi level. Fig. 4(a) shows the data recorded at $T=300 \mathrm{~K}$ at the incident photon energy of $68 \mathrm{eV}$. The observed features have been fitted by Gaussian peak shapes as shown in Fig. 4(a). The background is corrected by using Shirley method. The different features are due to the dominating $3 d$ band of elemental $\mathrm{Cu}$. To further study the valance band, we performed resonating photoemission spectroscopy in the incident photon energy range of 60 to $76 \mathrm{eV}$, which covers the $\mathrm{Cu} 3 p \rightarrow 3 d$ excitation energy and shows maximum at $\mathrm{E}=70 \mathrm{eV}$. Features centered around 0.8 and $2.5 \mathrm{eV}$ are mainly our region of interest, as it is dominated by the $\mathrm{Cu} 4 s$ and $3 d$ hybridized band, as observed in our DFT calculated density of states (DoS), as well as previous literature [18]. The photon energy dependence of spectral intensity of the features is shown in Figs. 4(b) and 4(c), which are known as constant initial state (CIS) plot. It is obtained from the area under the curve of different spectral features. The CIS of the feature at $0.8 \mathrm{eV}$ does not show any significant variation with the incident photon energy and is probably dominated by the $4 s$ orbital as the photoionization cross-section of $\mathrm{Cu} 4 s$ is much less than the $3 d$ orbital [19]. The feature at $2.5 \mathrm{eV}$ monotonically enhances with the increase in incident energy and shows strong resonance at around $70 \mathrm{eV}$. Here. the resonance occurs due to the quantum-mechanical interference between direct photoemission from $3 d$ and intra-atomic excitation of $3 p-3 d$ followed by super Coster-Kronig decay which is shown below.

$$
\begin{gathered}
3 p^{6} 3 d^{9} 4 s^{2}+h \nu \rightarrow 3 p^{6} 3 d^{8} 4 s^{2}+e^{-} \\
3 p^{6} 3 d^{9} 4 s^{2}+h \nu \rightarrow\left(3 p^{5} 3 d^{1} 04 s^{2}\right)^{*} \rightarrow 3 p^{6} 3 d^{8} 4 s^{2}+e^{-}
\end{gathered}
$$

The resonance enhancement near $\mathrm{Cu}: 3 p \rightarrow 3 d$ excitation energy indicates the presence of 3 d hole i.e. $3 d^{9} 4 s^{2}$ valence state of $\mathrm{Cu}$. The population of this state should reduce with the decrease in temperature due to reduced thermal energy. Therefore, the relative resonance intensity of the feature should be suppressed with the lowering of temperature. To confirm this, we have carried out RPES measurements at $T=170 \mathrm{~K}$, also shown in Fig. 4(c). We observe that the width of the resonance, which is prominently observed in feature B is reduced at $T=170 \mathrm{~K}$ relative to $300 \mathrm{~K}$. It indicates that the bandwidth corresponding to the $3 d-4 s$ overlapping has reduced at lower temperature, which is a manifestation of the reduced $3 d^{9} 4 s^{2}$ configuration. Therefore it confirms our earlier hypothesis. 


\section{SUMMARY:}

The temperature and magnetic field dependence of dc susceptibility of elemental $\mathrm{Cu}$ show dominant diamagnetism and an additional Curie-Weiss like behavior at low temperatures. The calculated dc susceptibility based on text book formula for orbital diamagnetism and Pauli paramagnetism is insufficient to explain the magnetic behavior in the measured range of temperature. DFT results indicate presence of small but significant contributions from $4 s$ orbital of $\mathrm{Cu}$. Based on these results, we propose a new mechanism involving fluctuation between two electronic states of copper. This leads to competition between different magnetic contributions due to the presence of holes in the $3 d$ band, which is further confirmed by RPES experiments. We conclude that the anomalous Curie-Weiss like paramagnetic behavior arises due to the presence of hole in certain fraction of $\mathrm{Cu}$ atoms, which is excited by thermal energy.

SP acknowledge Dr. L. S. Sarath Chandra and Md Akhlaq for help in analyzing the XRF data.

[1] Stefan Lebernegg, Alexander A. Tsirlin, Oleg Janson, and Helge Rosner, Phys. Rev. B 88, $224406(2013)$.

[2] Yingying Tang, Cheng Peng, Wenbin Guo, Jun-feng Wang, Gang Su, and Zhangzhen He, J. Am. Chem. Soc. 139, 14057 (2017).

[3] Meiyan Cui, Zhangzhen He, Nannan Wang, Yingying Tang, Wenbin Guo, Suyun Zhang, Lin Wang and Hongping Xiang, Dalton Trans. 45, 5234 (2016).

[4] S. Das, X. Zong, A. Niazi, A. Ellern, J. Q. Yan, and D. C. Johnston, Phys. Rev. B 76, 054418 (2007).

[5] Chien-Kang Hsu, Daniel Hsu, Chun-Ming Wu, Chi-Yen Li, Chi-Hang Hung, Chi-Hung Lee, and Wen-Hsien L, J. Appl. Phys. 109, 07 B528 (2011).

[6] Rainer Schmidt, J. Wu, C. Leighton, and I. Terry, Phys. Rev. B 79, 125105 (2007).

[7] J.-Q. Yan, J.-S. Zhou, and J. B. Goodenough, Phys. Rev. B 70, 014402 (2004).

[8] D. Phelanet al, Phys. Rev. Lett 96, 027201 (2006). 
[9] S. Kundu et al, Phys. Rev. Lett 125, 267202 (2020).

[10] Ahmad Ikhwan Us Saleheen, Ramakanta Chapai, Lingyi Xing, Roshan Nepal, Dongliang Gong, Xin Gui, Weiwei Xie, David P. Young, E. W. Plummer and Rongying Jin, npj Quantum Materials (2020) 5:53.

[11] Neil W. Ashcroft and N. David Mermin, Solid State Physics (Cengage Learning).

[12] J. M. D. Coey, Magnetism and Magnetic materials (Cambridge University Press).

[13] Stephen Blundell, Magnetism in Condensed Matter (Oxford University Press).

[14] Raymond Bowers, Phys. Rev. 102, 1486 (1965).

[15] F. Bitter, A. R. Kaufmann, C. Starr and S. T. Pan, Phys. Rev. 60, 134 (1941).

[16] G. Kresse and J. Furthmüller, Phys. Rev. B 54, 11169 (1996), G. Kresse and D. Joubert, ibid. 59, 1758 (1999).

[17] See the supplementary material.

[18] Abdesslem Jedidi, Shahid Rasul, Dilshad Masih, Luigi Cavallo and Kazuhiro Takanabe, J. Mater. Chem. A 3, 19085 (2015).

[19] J. J. Yeh and I. Lindau, At. Data Nucl. Data Tables 32, 1 (1985). 